\title{
Effects of PP modification and processing time on fiber/matrix interfacial strength for carbon fiber reinforced polypropylene
}

\author{
K. Tanaka, K. Ohno \& T. Katayama \\ Department of Biomedical Engineering, Doshisha University, Japan
}

\begin{abstract}
Polypropylene (PP) resin is expected to be used as the matrix of Carbon Fiber Reinforced Thermoplastics (CFRTP) because of its superior properties such as low cost and good processability. However, PP has poor interfacial adhesion with carbon fibers because PP is a non-polar polymer. Therefore, it is necessary to improve its interfacial properties in order to use PP as the matrix of CFRTP. The interfacial properties of CF/PP composites are reported to be improved by introducing a chemical reaction using a modified PP matrix. To enhance this chemical reaction which is time dependent, the processing time is likely to affect the interfacial strength. In this study, to evaluate the influence of PP modification and processing time on the fiber/matrix interfacial shear strength of CF/PP model composites, single fiber pull-out tests were carried out on $\mathrm{CF} / \mathrm{PP}$ composites produced at different processing times using unmodified PP and various modified $\mathrm{PP}$. The interfacial shear strengths of $\mathrm{CF} / \mathrm{PP}$ composites using modified PP were higher than those of CF/PP composites using unmodified PP. Long processing time improved the interfacial strength regardless of PP modification and the highly polar modified PP had the highest improve ratio of the interfacial strength.
\end{abstract}

Keywords: carbon fiber, polypropylene, PP modification, interfacial properties, processing time.

\section{Introduction}

Recently, the reduction of carbon dioxide emissions in the automotive industry has been required by the growing interest in environmental issues. The carbon dioxide emission is correlated to the weight of a car, so it is necessary to reduce the weight of cars in order to improve their fuel efficiency [1]. Therefore, Carbon Fiber 
Reinforced Plastics (CFRP), which have excellent mechanical properties such as high specific stiffness and strength, are anticipated to be applied to mass-produced vehicles [2]. Although the aerospace industry is mainly using epoxy resins for the matrix of CFRP [3], thermoplastics are desirable for the automobile industry due to their high processability and recyclability [4]. Among various thermoplastics, Polypropylene (PP) resin is expected to be used as the matrix of CFRTP because of its superior properties such as low cost and good processability [5]. However, as PP is a polyolefin resin and do not have polarity, PP has poor interfacial adhesion with fibers [6]. Therefore, it is necessary to improve their interfacial properties in order to use PP as the matrix of CFRTP. In the previous studies, as one of the methods of improving the interfacial properties between carbon fiber and PP, the addition of a modified PP such as maleic anhydride modified PP in $\mathrm{CF} / \mathrm{PP}$ composites has been reported [7-9]. In these papers, the interfacial properties of CF/PP composites are improved by introducing a chemical reaction using a modified PP matrix having polar functional groups. To enhance chemical reaction which is time dependent, the processing time is likely to affect the interfacial strength. However, the effect of processing time on the interfacial properties of $\mathrm{CF} / \mathrm{PP}$ composites has not been clarified. In this study, the fiber/matrix interfacial shear strength of CF/PP model composites, which were produced at different processing times using unmodified PP and various modified PP, were evaluated by single fiber pull-out tests, and the effects of PP modification and processing time on fiber/matrix interfacial strength were clarified.

\section{Materials and experimental procedure}

\subsection{Materials and specimen preparation}

PAN-based carbon fibers were used for single fiber pull-out tests. Unmodified PP pellets, medium polar modified PP pellets, and highly polar modified PP pellets were used for the matrix. Figure 1 shows a schematic drawing of the preparation of a fiber pull-out test specimen. Polyester thin film was used for a tab. After a single fiber was glued to the tab with adhesive, the tab was attached to a micromanipulator. The respective resin pellets were placed on the aluminum

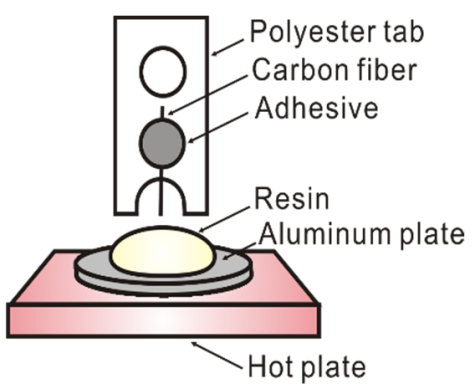

Figure 1: Preparation of a fiber pull-out test specimen. 
plate heated to $190^{\circ} \mathrm{C}$. After the carbon fiber was inserted to the designed depth in melted resin, the power supply to the hot plate was turned off and the specimen was air-cooled. The processing time is defined as the time range from the finishing the insert of the fiber into melted resin to turning off the power of the hot plate. The specimens using each PP pellets were made at processing times of 0 seconds or 300 seconds. In addition, the specimens using highly polar modified PP were made at a processing time of 60 seconds, 180 seconds and 600 seconds.

\subsection{Single fiber pull-out tests}

The single fiber pull-out tests were conducted using a testing machine for micro material (MMT-11N Shimadzu Co., Japan, Load capacity: $2.5 \mathrm{~N}$ ) with a constant displacement rate of $1.67 \times 10^{-6} \mathrm{~mm} / \mathrm{s}(0.1 \mathrm{~mm} / \mathrm{min})$. After chucking the polyester tab of the pull-out specimen, the supporting part of the tab was cut and a load was applied to the carbon fiber. Embedded fiber lengths and fiber diameters were measured using SEM after the pull-out tests.

\section{Results and discussions}

Figure 2 shows the relationship between embedded fiber lengths and maximum loads obtained by the single fiber pull-out tests of CF/PP composites using each PP matrix. Figure 3 shows the CF/PP interfacial shear strength, calculated by the following equation.

$$
\tau=\frac{F_{\max }}{\pi d l}
$$

where $F_{\text {max }}$ represents the maximum load required to pull-out the carbon fiber from the resin, $d$ is the fiber diameter and $l$ is the embedded fiber length.

In the case of 0 seconds of processing time, the interfacial shear strengths of $\mathrm{CF} /$ unmodified $\mathrm{PP}, \mathrm{CF} /$ medium polar modified $\mathrm{PP}$ and $\mathrm{CF} /$ highly polar modified PP were 7.52 $\mathrm{MPa}, 10.8 \mathrm{MPa}$ and $15.9 \mathrm{MPa}$, respectively. The interfacial shear strengths of $\mathrm{CF} /$ medium polar modified $\mathrm{PP}$ and $\mathrm{CF} /$ highly polar modified PP were higher than those of CF/unmodified PP by $44 \%$ and $110 \%$, respectively. Therefore, the interfacial shear strengths of CF/PP composites using modified PP were higher than those of CF/PP composites using unmodified PP. These results indicate that the PP modification has strongly influenced the interfacial properties.

In the case of 300 seconds of processing time, the interfacial shear strengths of $\mathrm{CF}$ /unmodified $\mathrm{PP}, \mathrm{CF} /$ medium polar modified $\mathrm{PP}$ and $\mathrm{CF} /$ highly polar modified $\mathrm{PP}$ were $11.3 \mathrm{MPa}, 13.5 \mathrm{MPa}$ and $31.9 \mathrm{MPa}$, respectively. When processing time was 300 seconds, the interfacial shear strengths of CF/unmodified PP, CF/medium polar modified PP and CF/highly polar modified PP were higher than those in the case of 0 seconds of processing time by $50 \%, 25 \%$ and $100 \%$, respectively. Long processing time improved the interfacial strength regardless of PP modification and the ratio of increasing the interfacial strength was highest in the case of using highly polar modified PP. These results suggest that the highly polar modified PP enhances the chemical reaction with long processing time, thus improving the $\mathrm{CF} / \mathrm{PP}$ interfacial properties. 


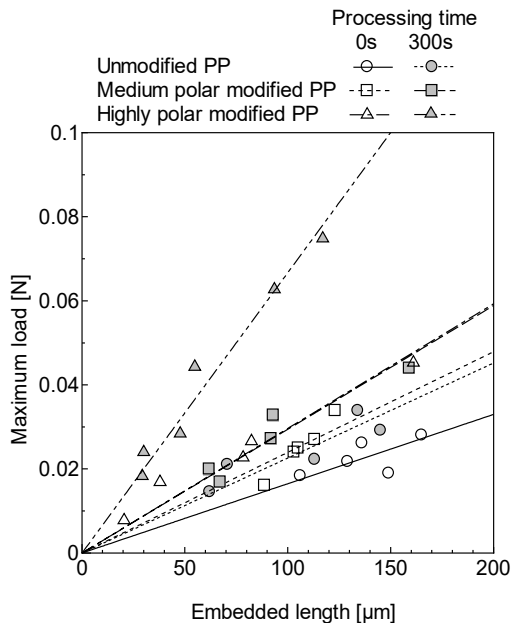

Figure 2: Relationship between embedded fiber lengths and maximum loads of $\mathrm{CF} / \mathrm{PP}$ model composites.

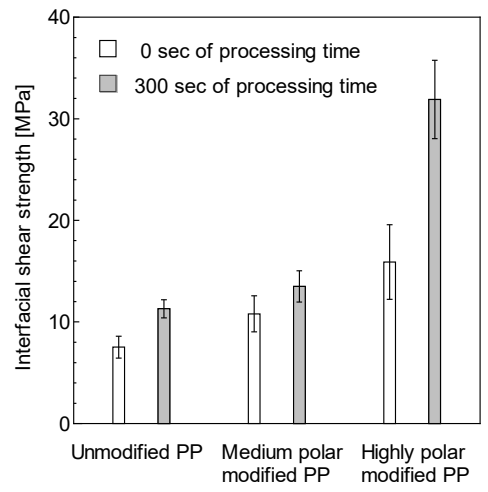

Figure 3: $\quad \mathrm{CF} / \mathrm{PP}$ interfacial shear strengths.

Figure 4 shows the relationship between embedded fiber lengths and maximum loads obtained by the single fiber pull-out tests of CF/highly polar modified PP composites at different processing times. Figure 5 shows the $\mathrm{CF} /$ highly polar modified PP interfacial shear strengths, calculated by the equation (1). Before 300 seconds, the interfacial shear strength showed an increasing tendency when processing time increased. However, the interfacial shear strength at 600 seconds of processing time was almost the same as that of 300 seconds. These results suggest that the interfacial shear strength gets saturated with a certain processing time and 300 seconds is optimum processing time in this case. 


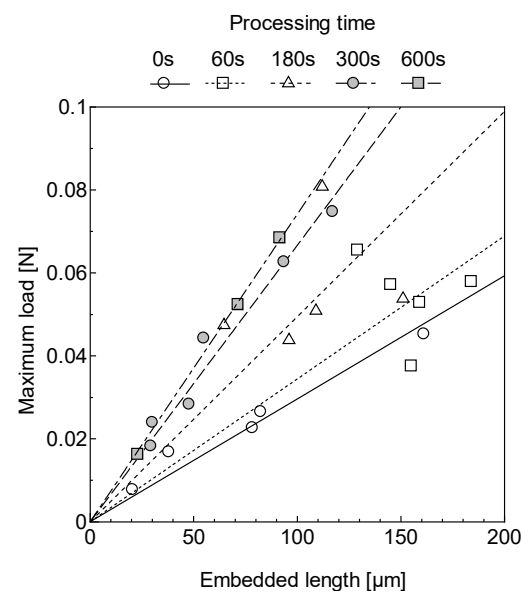

Figure 4: Relationship between embedded fiber lengths and maximum loads of $\mathrm{CF} /$ highly polar modified PP composites.

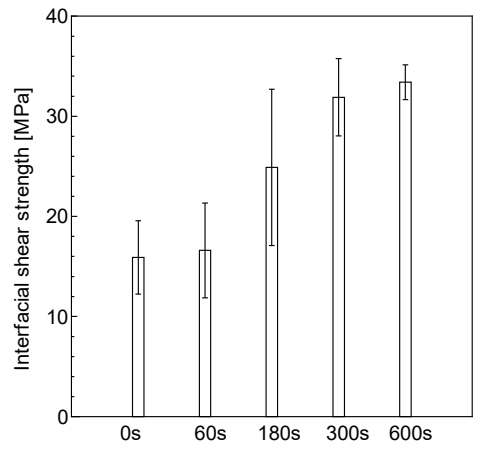

Figure 5: $\quad \mathrm{CF} /$ highly polar modified PP interfacial shear strengths.

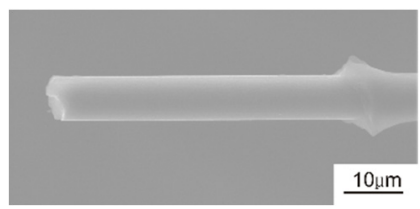

Figure 6: Pull-out fiber for CF/highly polar modified PP at 300 seconds of processing time.

Figure 6 shows a typical fiber surface of the pull-out specimen for CF/highly polar modified PP at 300 seconds of processing time. The fiber surface looked smooth and meniscus was observed. Regardless of PP modification or processing time, fiber surfaces for other specimens looked smooth as shown in Figure 6. 


\section{Conclusion}

In this study, to evaluate the influence of PP modification and processing time on the fiber/matrix interfacial shear strength of CF/PP model composites, single fiber pull-out tests were carried out on $\mathrm{CF} / \mathrm{PP}$ composites produced at different processing times using unmodified PP and various modified PP. The investigation yielded the following conclusions:

(1) Interfacial shear strengths of $\mathrm{CF} / \mathrm{PP}$ composites using modified PP were higher than those of CF/PP composites using unmodified PP, and this indicates that the PP modification has strongly influenced the interfacial properties.

(2) Long processing time improved the interfacial strength regardless of PP modification and the highly polar modified PP had the highest improve ratio of the interfacial strength.

\section{References}

[1] Composites penetration growth in Automotive: towards mass production 2010-2020 trends and forecasts, JEC Composites, pp. 13-22, 2011.

[2] R. Stewart, New developments help composites compete, Reinforced plastics, Vol. 47, No. 2, pp. 27-31, 2003.

[3] C. Soutis, Carbon fiber reinforced plastics in aircraft construction, Material Science and Engineering: A, Vol. 412, No. 1-2, pp. 171-176, 2005.

[4] A. Galoone, M. Ariante, G. Fusco, F. F. Flores, G. Bizzarro, A. Zinno \& A. Prota, Thermoplastic composite structure for mass transit vehicle: design, computational engineering and experimental validation, 15th European Conference on Composite Materials, pp. 24-28, 2012.

[5] F. Rezaei, R. Yunus \& N. A. Ibrahim, Development of short-carbon fiberreinforced polypropylene composite for car bonnet, Polymer-Plastic technology and Engineering, Vol. 47, No. 4, pp. 351-357, 2008.

[6] J. L. Thomason \& L. Yang, Temperature dependence of the interfacial shear strength in glass-fibre polypropylene composites, Composites Science and Technology, Vol. 71, pp. 1600-1605, 2011.

[7] S. Enoki, K. Kojima, S. Mizuno, K. Katayama \& K. Tanaka, High-speed compression molding of continuous carbon fiber reinforced polypropylene, WIT Transactions on The Built Environment, Vol. 137, pp. 311-315, 2014.

[8] Nevin Gamze Karsli \& Ayse Aytac, Effects of maleated polypropylene on the morphology, thermal and mechanical properties of short carbon fiber reinforced polypropylene composites, Materials and Design, Vol. 32, pp. 4069-4073, 2011.

[9] J. S. Kim and D. H. Kim, Compatibilizing effects of maleic anhydridegrafted-polypropylene (PP) on long carbon fiber-reinforced PP composites, Journal of Thermoplastic Composite Materials, pp. 1-13, 2014. 